IAU Colloquium 190 on Magnetic Cataclysmic Variables

ASP Conference Series, Vol. 315, 2004

Sonja Vrielmann 8 Mark Cropper, eds.

\title{
Spectroscopic Spin Variability in new IP Candidates
}

\author{
Domitilla de Martino \\ INAF - Astronomical Observatory of Capodimonte, Via Moiariello 16, \\ I-80131, Naples, Italy \\ Frank Haberl \\ Max Planck Institute for Extraterrestrial Physics, Giessenbachstrasse \\ D-85748 Garching, Germany \\ Juan M. Alcalá, Aniello Grado \\ INAF - Astronomical Observatory of Capodimonte, Via Moiariello 16, \\ I-80131, Naples, Italy
}

\begin{abstract}
We present fast spectroscopy of RX J1548-45 and BT Mon acquired with EFOSC-ESO $3.6 \mathrm{~m}$ in March \& April 2002, with the aim to detect for the first time spectral variability at the white dwarf rotation.
\end{abstract}

\section{RX J1548-45}

RX J1548-45 was recently identified as an IP from the presence of a X-ray and optical photometric period of $693 \mathrm{~s}$ (Haberl et al. 2002). Timing analysis of our fast spectrosocopy acquired in March (15 exposures of $120 \mathrm{~s}$ each; total time $0.6 \mathrm{~h}$ ), on April 28 (126 exposures of $90 \mathrm{~s}$ each; total time 4.4h) and April 29 (90 exposures of $90 \mathrm{~s}$ each; total time $2.9 \mathrm{~h}$ ) shows that radial velocities and EWs of emission lines of $\mathrm{HeII}, \mathrm{H}_{\gamma}, \mathrm{H}_{\beta}$ and $\mathrm{He} \mathrm{I}(\lambda \lambda 5875$ and 4471$)$ are also modulated at this period (Fig. 1). This definitively allows us to identify it as the spin period of the white dwarf (WD). Broad (FWHM $50 \AA$ ) absorption features are also detected around Balmer lines similar to those observed in V 709 Cas (BonnetBidaud et al. 2001), thus suggesting an origin in the WD photosphere. Their EWs are found to be weakly variable at the spin period. We have used DA WD models by Koester (2002), to estimate the temperature and gravity, but these result to be unconstrained due to the contamination of emissions in the Balmer lines. For $\log g=8.0, T>26000 \mathrm{~K}$. The observed variability and the high temperature favour an origin in the X-ray irradiated WD polar region.

\section{BT Mon}

BT Mon, a poorly observed eclipsing Old Nova, was included in the group of SW Sex stars for its emission line characteristics and behaviour at its long $(8.01 \mathrm{~h})$ orbital period. A magnetic nature was suggested by White et al. (1996). It was proposed as an IP candidate by Smith et al. (1998) from the detection of 

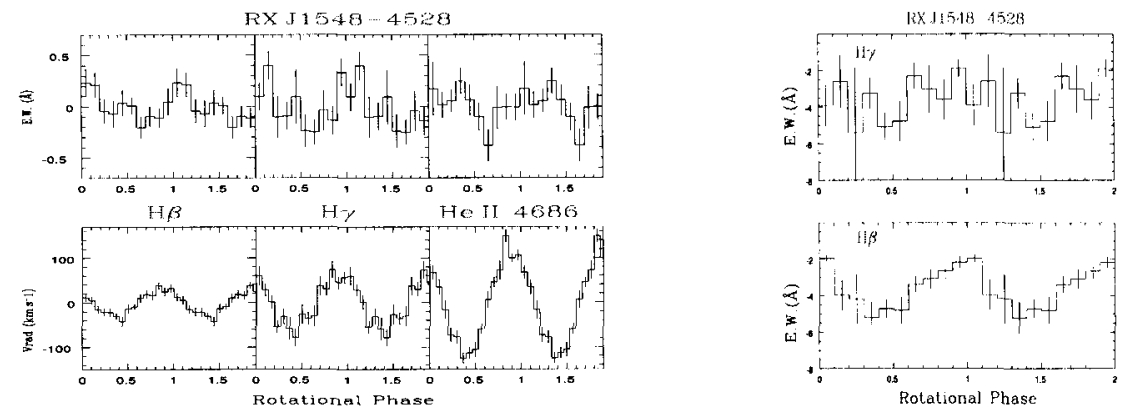

Figure 1. Left: Folded radial velocity (bottom) and EW (top) curves of $\mathrm{H}_{\beta}, \mathrm{H}_{\gamma}$ and $\mathrm{He}$ II at the $693 \mathrm{~s}$ period. Right: Folded EWs of Balmer absorptions.

a quasi-periodic flaring activity on a timescale of tens of minutes. From our short $(1.2 \mathrm{~h}$ ) observing run (31 spectra of $2 \mathrm{~m}$ exposure each), we have detected a variability in emission line radial velocities and EWs of $\mathrm{He} \mathrm{II}, \mathrm{HeI}, \mathrm{H}_{\beta}$ and $\mathrm{H}_{\gamma}$ as well as in the continuum at a $53 \pm 4 \mathrm{~m}$ period (Fig. 2). We interpret this as the WD spin period, thus supporting its IP nature. BT Mon will be the $9^{\text {th }}$ IP added to the small group of magnetic novae (see also Woudt \& Warner, these proceedings.).

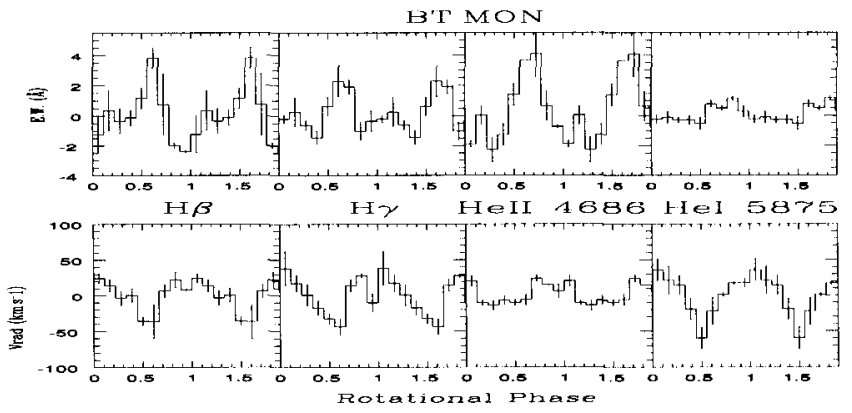

Figure 2. Folded radial velocity (bottom) and EW (top) curves of Balmer, $\mathrm{He}$ II and $\mathrm{HeI}$ emissions at the $53 \mathrm{~m}$ period.

\section{References}

Bonnet-Bidaud, J.M., Mouchet, M., de Martino, D., Matt, G., Motch, C. 2001, A\&A, 374, 1003

Haberl, F., Motch, C., \& Zickgraf, F.J. 2002, A\&A, 387, 201.

Koester D., 2002, private communication

Smith, D., Dhillon, V., \& Marsh, T. 1998, MNRAS, 296, 465.

White, J., Schlegel, E., \& Honeycutt, R.K. 1996, ApJ, 456, 777. 